\title{
Erratum to: On the Characterisations of a New Class of Strong Uniqueness Polynomials Generating Unique Range Sets
}

\author{
Abhijit Banerjee $^{1}$ • Sanjay Mallick ${ }^{1}$
}

Published online: 29 July 2017

(C) Springer-Verlag GmbH Germany 2017

\section{Erratum to: Comput. Methods Funct. Theory (2017) 17:19-45 DOI 10.1007/s40315-016-0174-y}

In the proof of Lemma 2.6 Case 2 has to be replaced as follows.

Case 2 Let $A=\omega^{l}$ for some $l$ such that $0 \leq l \leq m-1$. Then also $F\left(t_{0}\right)=0=F^{\prime}\left(t_{0}\right)$ implies $e^{n t_{0}}=A$ and $e^{m t_{0}}=1$. Now, if possible, suppose that there exist more than one $t_{0}$ such that $e^{m t_{0}}=1$ and $e^{n t_{0}}=A$, i.e., there exist $t_{0 p}, t_{0 q}$ with $e^{t_{0 p}} \neq e^{t_{0 q}}$ such that $e^{m t_{0 p}}=1=e^{m t_{0 q}}$ and $e^{n t_{0 p}}=A=e^{n t_{0 q}}$, i.e., $e^{m\left(t_{0 p}-t_{0 q}\right)}=1$ and $e^{n\left(t_{0 p}-t_{0 q}\right)}=1$, i.e., $m\left(t_{0 p}-t_{0 q}\right)=2 k_{1} \pi i$ for some $k_{1} \in \mathbb{Z}$ and $n\left(t_{0 p}-t_{0 q}\right)=2 k_{2} \pi i$ for some $k_{2} \in \mathbb{Z}$. Since $\operatorname{gcd}(m, n)=1$, so there exists $x, y \in \mathbb{Z}$ such that $m x+n y=1$, i.e., $m\left(t_{0 p}-t_{0 q}\right) x+n\left(t_{0 p}-t_{0 q}\right) y=\left(t_{0 p}-t_{0 q}\right)$, i.e., $2 k_{1} \pi i x+2 k_{2} \pi i y=\left(t_{0 p}-t_{0 q}\right)$, i.e., $2 \pi i\left(x k_{1}+y k_{2}\right)=\left(t_{0 p}-t_{0 q}\right)$, i.e., $2 s \pi i=\left(t_{0 p}-t_{0 q}\right)$, where $s=x k_{1}+y k_{2} \in \mathbb{Z}$.

Therefore $e^{t_{0 p}}=e^{t_{o q}}$, which is a contradiction to $e^{t_{0 p}} \neq e^{t_{0 q}}$. Therefore $\phi\left(e^{t}\right)$, hence $\phi(z)$, has exactly one multiple zero $\omega^{j}$, where $0 \leq j \leq m-1$ and $\omega^{m j}=1, \omega^{n j}=\omega^{l}$ and that is of multiplicity 4 . Now in particular if $A=1$, then we have $\omega^{j}$ is the multiple zero of $\phi(z)$ for some $j \in\{0,1, \ldots, m-1\}$ such that $\omega^{m j}=1$ and $\omega^{n j}=1$ i.e., $\omega^{j}=1 \operatorname{as} \operatorname{gcd}(m, n)=1$.

Communicated by Risto Korhonen.

The online version of the original article can be found under doi:10.1007/s40315-016-0174-y.

$凶$ Abhijit Banerjee

abanerjee_kal@yahoo.de; abanerjeekal@gmail.com

Sanjay Mallick

smallick.ku@gmail.com

1 Department of Mathematics, University of Kalyani, Kalyani, West Bengal 741235, India 